\title{
Protective effect of salvianolic acid B against intestinal ischemia reperfusion-induced injury in a rat model
}

\author{
Jiaxi Chen ${ }^{1 \star}$, Weiming Zhou ${ }^{2}$, Zhibin Zhou ${ }^{3}$, Tangzhan Yuan ${ }^{1}$, Bo Li ${ }^{1}$ and Yang \\ Zheng $^{1}$ \\ ${ }^{1}$ Department of General Surgery, The Fourth Affiliated Hospital of Nanchang University, NanChang, Jiangxi 330000, \\ ${ }^{2}$ Department of Vascular Surgery, The Second Affiliated Hospital of Nanchang University, NanChang, Jiangxi 330006, \\ ${ }^{3}$ Department of Research, The Fourth Affiliated Hospital of Nanchang University, NanChang, Jiangxi 330000, China
}

*For correspondence: Email: jaixichen@hotmail.com; Tel: 0086-79186663001

Sent for review: 7 May 2017

Revised accepted: 23 September 2017

\begin{abstract}
Purpose: To evaluate the gastro-protective efficacy of salvianolic acid B (SAB) against intestinal ischemic-reperfusion injury (IIRI) in a rat model.

Methods: Forty-eight healthy male rats were randomly choosen and divided into 4 groups of 12 rats each. Control group rats underwent laparotomy without occlusion; IIRI group rats underwent laparotomy with occlusion for $60 \mathrm{~min}$, followed by $24 \mathrm{~h}$ of reperfusion; SAB + IIRI group received 7 days of pretreatment with $40 \mathrm{mg} / \mathrm{kg}$ of $S A B+I I R /$; while the fourth group received only SAB. The antioxidant, inflammatory markers, intestinal permeability marker, as well as intestinal histopathological changes were assessed.

Results: The activities of antioxidants including reduced glutathione (GSH), catalase (CAT) and superoxide dismutase $(S O D)$ were significantly ameliorated $(p<0.01)$ in $S A B$-supplemented group

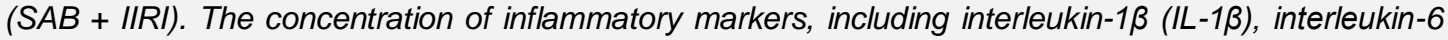
(IL-6), tumor necrosis factor alpha (TNF- $\alpha$ ) and nuclear factor p65 (NF-p65) as well as small intestinal permeability marker (FITC-Dextran), were significantly reduced $(p<0.01)$ following administration of $S A B$ for 7 days. In addition, pretreatment with SAB reverted intestinal (ileum) histopathological changes to almost normal architecture with significant reduction in Chiu score.

Conclusion: The results of this study demonstrate that $S A B$ may protect the intestine by attenuating oxidative stress and inflammatory response and hence, may be potentially for treating IIRI.
\end{abstract}

Keywords: Salvianolic acid B, Intestinal Ischemia-reperfusion, Antioxidants, Inflammation, Intestinal permeability

Tropical Journal of Pharmaceutical Research is indexed by Science Citation Index (SciSearch), Scopus, International Pharmaceutical Abstract, Chemical Abstracts, Embase, Index Copernicus, EBSCO, African Index Medicus, JournalSeek, Journal Citation Reports/Science Edition, Directory of Open Access Journals (DOAJ), African Journal Online, Bioline International, Open-J-Gate and Pharmacy Abstracts

\section{INTRODUCTION}

Intestinal ischemia-reperfusion injury (IIRI) is a serious clinical condition occurred during septic shock, cardiopulmonary bypass, small bowel transplantation, strangulated hernias due to gastric barrier dysfunction and bacterial translocation [1]. During intestinal ischemia, rapid restoration of blood flow is vital, nevertheless reperfusion might collapse the systemic circulation that triggers systemic inflammatory response and mucosal injury and ultimately ends up in multi-organ failure (MOF) and thus entails with increased mortality and morbidity rate [2]. Several researchers have indicated that inflammatory response and oxidative stress are the two crucial events that act as vicious cycle and elicit mucosal damage and ultimately results in IIRI $[3,4]$. Previously, studies have highlighted that phytocompounds with antioxidant and anti- 
inflammatory properties abolish IIRI in various animal models $[5,6]$.

Salvianolic acid B (SAB), a natural water-soluble phenolic acid extracted from Salvia militiorrhiza (Danshen), is prescribed for improving blood circulation related to menstrual and dermal disorders [7]. SAB also exert various pharmacological properties including antioxidant, anti-cancer, anti-inflammatory and immunomodulatory [8,9]. Previously, Salvia militiorrhiza has shown to protect gastric mucosa owing to the presence of salvianolic acid $A / B$ [10]. Moreover, SAB has a positive effect on various experimental IR model in different organs $[7,11,12]$. Hence, it is probable that SAB may also alleviate IR-induced injury in rat model. Therefore, this pre-clinical study was aimed to explore the gastroprotective property of SAB by evaluating various inflammatory markers (cytokines), antioxidant status and ileum histopathology analysis in IIRI a rat model.

\section{EXPERIMENTAL}

\section{Experimental rats}

Male healthy Sprague-Dawley rats $(n=48)$ weighing $280 \pm 10 \mathrm{~g}$ were procured from Animal center of Nanchang University, China. All the animals were maintained at $22 \pm 2{ }^{\circ} \mathrm{C}$ with $12 \mathrm{~h}$ day light/ night dark cycle with full access to food and water (ad libitum) in a stainless-steel cage. The Institutional Ethical Committee Board have approved this experimental study/protocol (NCU no. 8305-16) and was performed at the Animal Center of Nanchang University following the guidelines of the United States National Institutes of Health (NIH) [13].

\section{Surgical procedure/IRII insult}

All the rats were fasted for $12 \mathrm{~h}$ and anesthetized using pentabarbital sodium at the dose of 50 $\mathrm{mg} / \mathrm{kg}$ through via intraperitoneal (i.p) injection. Rats were placed on a warm pad to maintain at $37^{\circ} \mathrm{C}$ throughout the surgical protocol. The intestinal IR injury was induced by performing midline laparotomy followed by isolation of the superior mesenteric artery (SMA) and followed by clamping by atraumatic arterial clamp for 60 min to block splanchnic circulation. Ischemia was confirmed by the absence of pulsation as well as pale color appearance. Then, reperfusion was performed by removing the clamp, and the reperfusion was confirmed by the recurrence of the pulse as well as the pinkish color appearance of the intestine. The surgical wound was closed using nylon sutures and reverted to respective cage. All the experimental rats underwent midline laparotomy followed by occlusion and reperfusion except the control rats which were not clamped.

\section{Animal groups}

Forty-eight healthy male SD rats were divided into 4 groups with 12 rats in each. Group I rats underwent laparotomy without occlusion or clamping and received only saline and served as control group. Group II rats underwent laparotomy with occlussion for $60 \mathrm{~min}$ and followed by $24 \mathrm{~h}$ of reperfusion (as indicated above) and received only saline and served as IIRI group. Group II rats were pretreated with 40 $\mathrm{mg} / \mathrm{kg}$ of SAB (based on prelimineary studies) via i.p. by dissolving with saline for 6 days prior IIR induction and $1 \mathrm{~h}$ prior IIRI induction and served as $S A B$ + IIRI group, Group IV rats underwent laparotomy without occlusion received only SAB for 7 days and served as SAB alone group.

\section{Sample preparation}

Rats were sacrificed by cervical decapitation under pentabarbotal sodium at the dose of 50 $\mathrm{mg} / \mathrm{kg}$ via i.p after IIRI induction. Blood samples were collected in a heparinized tube to separated plasma. The small intestine-ileal tissues $(20 \mathrm{~cm})$ were excised immediately and a portion of ileal tissue $(5 \mathrm{~cm})$ was fixed in $10 \%$ formalin for histological analysis and the remaining ilial tissues are homogenized (10 \%) using phosphate buffered saline (PBS) solution to yield ileal tissue lysate (supernatant), were used for various biochemical analysis.

\section{Determination of lipid peroxidation products and antioxidant activity}

Lipid peroxidation product like melondialdehyde (MDA), as well as antioxidant activities of reduced glutathione (GSH), catalase (CAT) and superoxide dismutase (SOD) were assessed in ileal lysate using kit from Nanjing Bioengineering Institute (Nanjing, China). One Unit of SOD activity is equalent to the amount required to inhibit $50 \%$ of auto-oxidation. One Unit $(U)$ of CAT One unit was defiend as $\mathrm{mg}$ of tissue protein that consumed $1 \mu \mathrm{mol} \mathrm{H}_{2} \mathrm{O}_{2}$ at $405 \mathrm{~nm}$ in $1 \mathrm{~s}$.

\section{Evaluation of myeloperoxidase (MPO) activity and proinflammatory cytokines}

Neutrophil infiltration was determined in the form of myeloperoxidase (MPO) activity in ileal lysate by MPO assay kit from Nanjing Bioengineering Institute (Nanjing, China). One Unit (U) of MPO 
activity was equal to the amount required for degrading $1 \mathrm{mmol}$ peroxidase $/ \mathrm{min}$ at $25^{\circ} \mathrm{C}$ is expressed as unit per $g$ wet weight $(\mathrm{U} / \mathrm{g})$. Moreover, nuclear factor kappa B (NF-kB) subunit (NF-p65) in the ileal tissue lysate were evaluated using nuclear fraction (extracted by NR-PER nuclear/cytoplasmic extract reagent from Thermo Fisher Scientific, MA, USA) and evlauated by NF-KB p65 transciption factor assay kit (Thermo Fisher Scientific, MA, USA). Furthermore, the levels of various proinflammatory cytokines such as interleukins- $1 \beta$ (IL-1ß), interleukins-6 (IL-6), and tumor necrosis factor alpha (TNF- $\alpha$ ) were measured using commercial ELISA kit (USCN Business Co., Ltd. Wuhan, China).

\section{Intestinal permeability assay}

To assess the intestinal permeability (epithelial barrier) of ileal tissue, fluorescein isothiocyanate (FITC)-conjugated dextran assay procedure was used by following the methods of Chang and his coworkers with slight modification [14]. Shortly, 1 $\mathrm{h}$ before the sacrifice, rats were anaesthetized under pentobarbital sodium at the dose of 50 $\mathrm{mg} / \mathrm{kg}$ via i.p and ileum region $(10 \mathrm{~cm})$ were clamped again and administered the solution of FD4 dissolved in PBS $(0.5 \mathrm{mg} / \mathrm{mL})$. After $1 \mathrm{~h}$ all the rats were euthanized, and a blood sample was collected via cardiac puncture and the fluorescence concentration (FD4) of the plasma was measured by microplate reader with an excitation/emission wavelength $485 / 528 \mathrm{~nm}$ with FITC standards. The concentration of FITCdextran was calculated using a standard curve and expressed as $\mu \mathrm{g} / \mathrm{mL}$.

\section{Histopathological studies}

Freshly isolated ileum tissue $(5 \mathrm{~cm})$ were cut down to small pieces and fixed in formaldehyde $(10 \%)$ for $10 \mathrm{~h}$ and embedded in paraffin wax to form a tissue block. Those blocks were sliced into $4 \mu \mathrm{m}$ sections and transferred to a glass slide and followed by staining with $\mathrm{H}$ \& $\mathrm{E}$ stain. The stained slide was covered with cover slip and viewed under a light microscope (OlympusBX53 Tokyo, Japan) at $400 \times$ for any histopathological changes. The degree of histopathological changes of the ileal section was graded according to Chiu scoring system based on Chiu method [15].

\section{Data analysis}

For data statistical analysis SPSS software (version 21, IBM Inc, NY, USA). Values are expressed as the mean \pm standard deviation (SD). Chiu score was analyzed with KruskalWallis test. For other parameters, the significance difference between the control group and $S A B$ group were analyzed using one-way ANOVA followed by LSD post-hoc test. were determined by one-way ANOVA followed by least significant difference (LSD) for multiple comparison. $P<0.05$ was considered statistically significant.

\section{RESULTS}

Effect of SAB on the activities of ileal antioxidants and lipid peroxidation products

The activities of ileal antioxidants and lipid peroxidation products in experimental rats were represented in Table 1. The activities of GSH, CAT and SOD were significantly abolished $(p<$ 0.01 ) with increased lipid peroxidation in IIR induced rats compared with control rats. Whereas, pretreatment with SAB for 7 days in IR-induced rats could significantly improve $(p<$ 0.01 ) the antioxidants activity with decreased lipid peroxidation product.

\section{Effect of SAB on ileal MPO levels}

Figure 1 illustrate the effect of SAB on ileal MPO levels in experimental rats. Rats in the IIRI group showed increase $(p<0.01)$ levels of MPO than control rats. Administration of $40 \mathrm{mg}$ of $\mathrm{SAB}$ in IIRI induced rats could considerably decreased $(p<0.01)$ the MPO levels than IRII rats.

Table 1: Effect of SAB on the activities of ileal antioxidants and lipid peroxidation products in experimental rats

\begin{tabular}{lcccc}
\hline Group & $\begin{array}{c}\text { CAT }(U / m g \\
\text { Protein) }\end{array}$ & $\begin{array}{c}\text { SOD }(\mathbf{m} / \mathbf{m g} \\
\text { Protein) }\end{array}$ & $\begin{array}{c}\text { GSH }(\mathbf{U} / \mathbf{m g} \\
\text { Protein) }\end{array}$ & $\begin{array}{c}\text { MDA } \\
\text { (nmol/mg } \\
\text { Protein) }\end{array}$ \\
\hline Control & $40.31 \pm 5.10$ & $5.03 \pm 0.44$ & $1.15 \pm 0.20$ & $1.79 \pm 0.12$ \\
IIRI & $28.33 \pm 4.00 \mathrm{a}^{\star *}$ & $3.78 \pm 0.27 \mathrm{a}^{\star *}$ & $0.71 \pm 0.12 \mathrm{a}^{\star *}$ & $2.34 \pm 0.10 \mathrm{a}^{\star *}$ \\
SAB + IIRI & $36.67 \pm 4.09 \mathrm{~b}^{* *}$ & $4.51 \pm 0.55 \mathrm{~b}^{\star *}$ & $0.95 \pm 0.09 \mathrm{~b}^{\star *}$ & $1.95 \pm 0.09 \mathrm{~b}^{\star *}$ \\
SAB alone & $39.34 \pm 6.23$ & $4.88 \pm 0.62$ & $1.08 \pm 0.11$ & $1.82 \pm 0.07$ \\
\hline
\end{tabular}

Values are expressed as the mean \pm standard deviation. ${ }^{* *} p<0.05$ (a) IIRI group compared with control rats, (b) $S A B+I I R I$ compared with IIRI group 


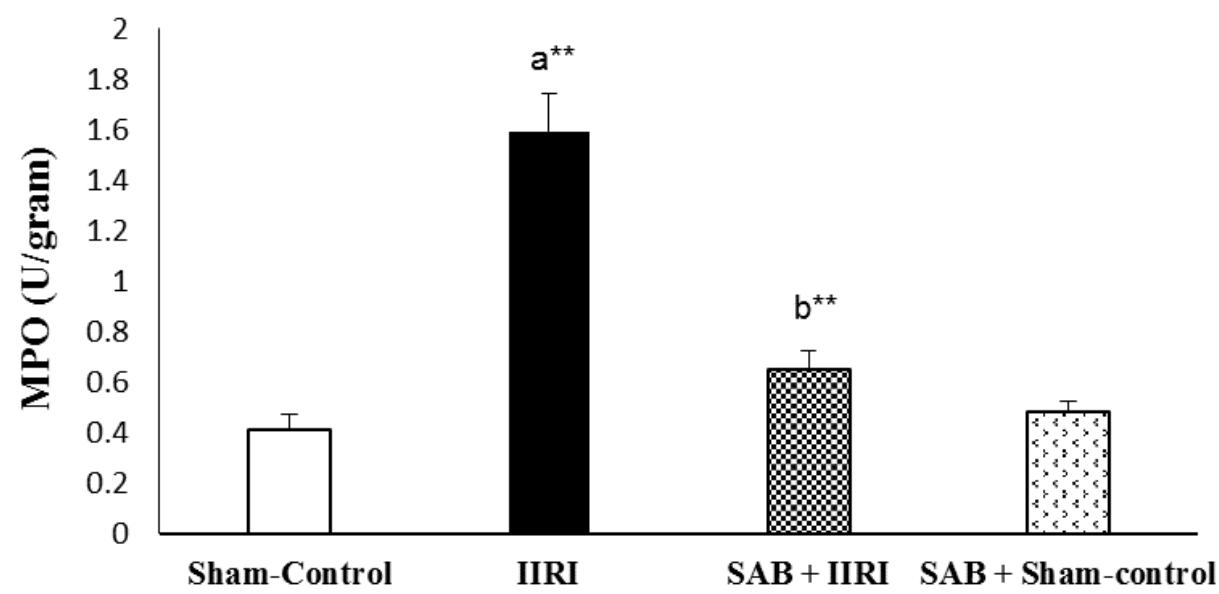

Figure 1: Effect of SAB on ileal MPO levels of experimental rats. Values are expressed as the mean \pm standard deviation; ${ }^{* *} p<0.05$ (a) IIRI group compared with control rats; (b) SAB + IIRI compared with IIRI group

\section{Effect of SAB on levels ileal inflammatory markers}

Table 2 epitomized the effect of SAB on the concentration of ileal inflammatory markers in experimental rats. The concentration of NF-p65 subunit, IL-1 $\beta, \mathrm{IL}-6$, and TNF- $\alpha$ in the ileal tissue of IIR induced rats were markedly increased $(p<$ 0.01 ). $S A B$ supplementation significantly decreased $(p<0.01)$ the concentration of NFp65 subunit, IL-1 $\beta$, IL-6, and TNF- $\alpha$ as compared to IIR group.

\section{Effect of SAB on small intestine permeability}

The effect of SAB on small intestinal permeability (ileum) in experimental rats are showed in Figure 2. A pronounced increase $(p<0.01)$ in the levels of FITC-Dextran (FD4) was observed in the intestinal IR-induced group. Treatment with SAB for 7 subsequent days prior to IIR induction, could significantly decreased $(p<0.01)$ the levels of FITC-dextran in comparison with IIR rats.

Table 2: Effect of SAB on the concentration of ileal inflammatory markers in experimental rats

\begin{tabular}{|c|c|c|c|c|}
\hline Group & $\begin{array}{c}\text { TNF- } \alpha \text { (ng/g } \\
\text { protein) }\end{array}$ & $\begin{array}{c}\text { IL-1 } \beta \text { (ng/g } \\
\text { protein) }\end{array}$ & IL-6 (pg/g protein) & $\begin{array}{c}\text { NF-p65 (ng/g } \\
\text { protein) }\end{array}$ \\
\hline Control & $72.66 \pm 7.21$ & $41.10 \pm 8.80$ & $46.05 \pm 4.67$ & $65.23 \pm 5.12$ \\
\hline IIRI & $165.03 \pm 12.57 a^{* *}$ & $78.44 \pm 10.16 \mathrm{a}^{\star *}$ & $92.70 \pm 10.21 \mathrm{a}^{\star *}$ & $121.47 \pm 13.39 a^{* \star}$ \\
\hline$S A B+I I R I$ & $126.56 \pm 15.20 \mathrm{~b}^{* *}$ & $52.26 \pm 7.31 \mathrm{~b}^{* *}$ & $60.82 \pm 11.60 \mathrm{~b}^{* *}$ & $81.05 \pm 9.27 b^{* \star}$ \\
\hline SAB alone & $93.72 \pm 10.11$ & $44.91 \pm 10.22$ & $48.40 \pm 10.10$ & $69.11 \pm 11.22$ \\
\hline
\end{tabular}

Values are expressed as mean \pm standard deviation; ${ }^{* *} p<0.05$ (a) IIRI group compared with control rats, (b) $S A B+I I R I$ compared with IIRI group

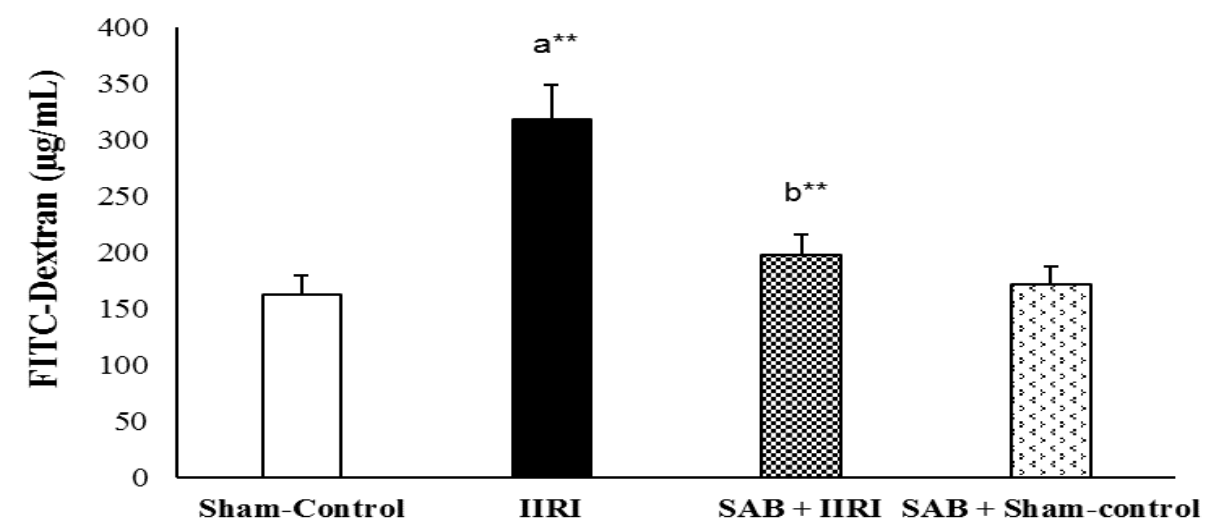

Figure 2: The effect of SAB on small intestinal permeability (ileum) in experimental rats. Values are expressed as the mean \pm standard deviation; ${ }^{* *} p<0.05$ (a) IIRI group compared with control rats, (b) SAB + IIRI compared with IIRI group 

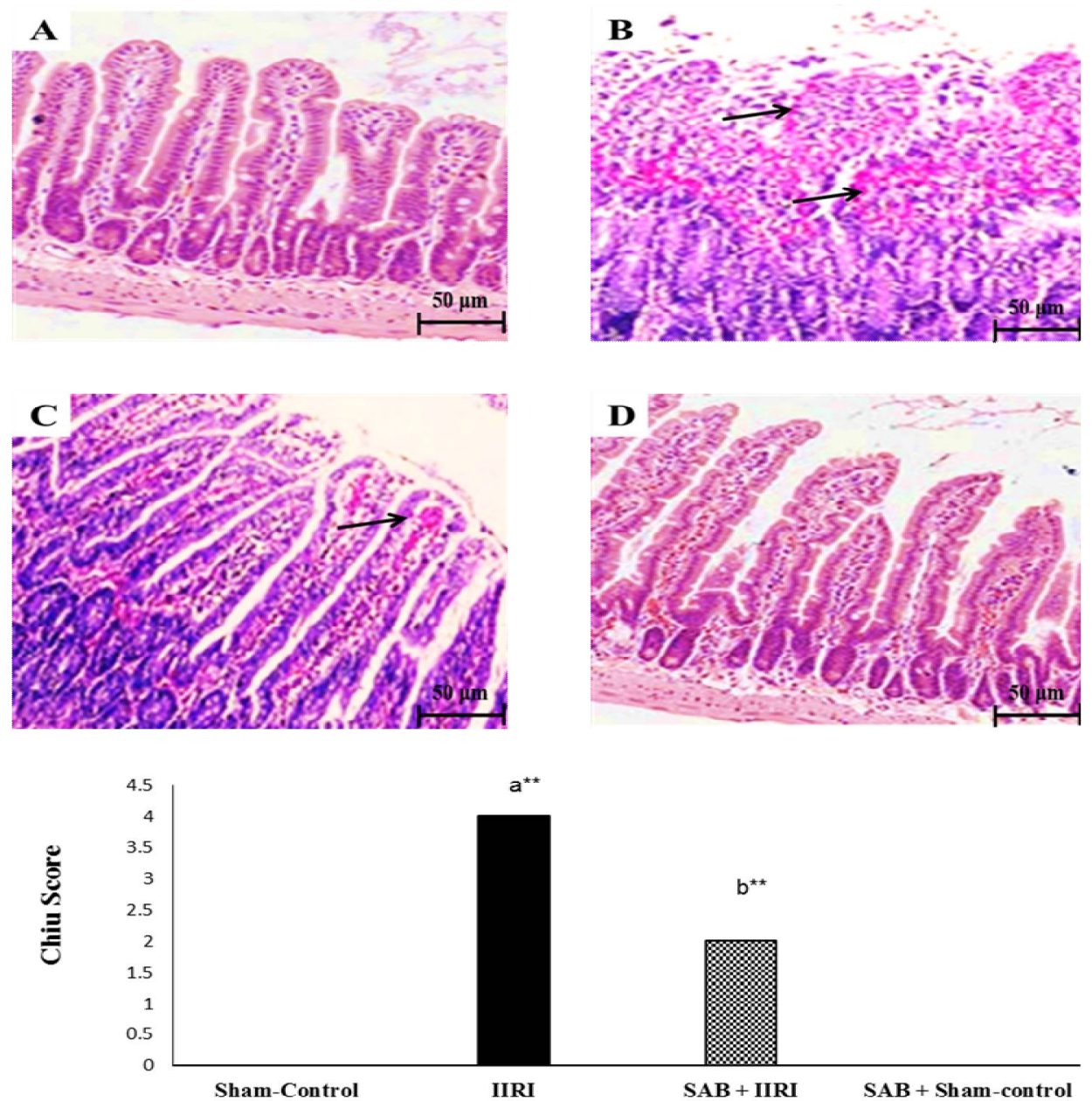

Figure 3: Effect of SAB on the ileal section with $\mathrm{H}$ \& $\mathrm{E}$ staining in experimental rats as well as Chiu score. The slide of the ileal tissue of control rats (nil Chiu score) showed the normal morphology/architecture of villus (Figure $3 A$ ). The slide of IIRI induced rats (highest Chiu score) represents the presence of denuded villi and lamina propria with massive infiltration of neutrophils and extensive necrosis (arrow mark) accompanied by disrupted brush border and edema (Figure 3B). Slide of SAB pretreated with IIRI induced rats (mild Chiu score) revealed a mild villi denudation with lesser edema and necrosis (arrow mark) (Figure 3C). Whereas, the slide of SAB + IIRI rats (nil Chiu score) displayed normal morphology of ileum with prominent villi and looked similar to control rats (Figure 3D); Values are expressed as the mean \pm standard deviation; ${ }^{* *} p<0.05$ (a) IIRI group compared with control rats, (b) SAB + IIRI compared with IIRI group

\section{Effect of SAB on histological changes in ileal tissue}

The histological changes in ileal tissue were evaluated using Chiu scores (Figure 3). The control rats ileal section showed normal mucosa with nil chiu score (Figure $3 \mathrm{~A}$ ). The ileal section of IIRI induced rats characterized by the presence of denuded villi and lamina propria with massive infiltration of neutrophils and extensive necrosis with highest $(p<0.01)$ Chiu score (Figure 3B). SAB pretreated with IIRI induced rats revealed a mild villi denudation with lesser edema and necrosis with lesser $(p<0.01)$ Chiu score (Figure $3 \mathrm{C}$ ). SAB alone treated rats showed normal morphology of ileum with zero Chiu score (Figure 3D).

\section{DISCUSSION}

The present study highlighted that IRII induced rats pretreated (preconditioned) with SAB for 7 days could significantly enhance the antioxidant status, with reduced inflammatory markers, intestinal permeability and histopathological changes and thus showcasing its gastroenteroprotective activity. Intestine and intestinal mucosa are highly susceptible to ROS (reactive oxygen species) after ischemia-reperfusion (IR) injury, as they act as intestinal barrier (prevent bacterial translocation) and directly involved in maintaining immune response as well as maintaining the nutritional homeostasis (exogenous antioxidants) by protecting enterocytes of villi [16]. 
In ischemia, lack of oxygen supply, would provoke the excessive production of ROS. Upon reperfusion, the ROS generation were enhanced and ultimately end in tissue or cell damage via lipid peroxidation [17] and hence the levels of MDA were increased with decreased antioxidant activities. Pretreatment with $\mathrm{SAB}$ significantly lowered the excessive production of free radicals in IR-induced rats. The results are in aggrement with the results of Liu et al [18], who also noticed that pretreatment with salvianolic acid $B$ could significantly increased the activities of GSH, CAT and SOD in the PC12 cell line model.

Tongqiang et al [19] demonstrated that salvianolic acid B could up-regulate Nrf2 (Nuclear factor erythroid-2-related factor) protein expression and thereby enhance the production of various endigenous antioxidants like SOD and GSH. Several researchers have indicated that two free hydroxyl groups of salvianolic acid B could contribute to its free radical quenching ability and thus concomitantly lowered the lipid peroxidation products production $[20,21]$.

Rats in the IIR group showed increased levels of MPO than control rats due to exuberant ROS production via activation of polymorphonuclear neutrophils (PNMs) and ultimately increases the neutrophil infiltration in the intestine and results in MOF. SAB administration in IIRI induced rats significantly lowered the MPO levels by inhibiting neutrophil infiltration to the small intestine and thus protect the ileum from further damage. Previously, salvianolic acid B has been shown to alleviate neutrophil infiltration (activation) in ischemic reperfusion rabbit model owing to its anti-inflammatory property [22].

The concentration of NF-p65 subunit, IL-1 $\beta$, IL-6, and TNF- $\alpha$ in the ileal tissue of IIR induced rats were significantly increased. As mentioned earlier, during IIR condition excessive activation of PNMs might upregulate the proinflammatory cytokines like IL-1 $\beta$, IL- 6 , and TNF- $\alpha$ via NF-KB signaling pathway [4]. $S A B$ intervention significantly brought back those inflammatory markers concentration to near normal level due to anti-inflammatory activity. Similar to our results, Wang and his colleague also documented that $S A B$ could substantially restrain the levels of IL-1 $1 \beta, I L-6$ and TNF- $\alpha$ by downregulating NF-KB via inhibiting a TLR4 signaling pathway in cerebral ischemia/reperfusion injury model [23].

A marked increase in the levels of FITC-dextran (FD4) was observed in IIRI group due to the disruption of the tight junction (loss of adhesion molecules) [24]. Treatment with SAB might have decreased the levels of FITC-dextran owing to its antioxidant and anti-inflammatory properties. Deng et al indicated that salvianolic acid B with ginsenoside (Rg1) downregulates cytokine expression such as TNF- $\alpha$, IL-1 $\beta$ and thus, improves the microvascular permeability in cardiac IR injury model [25].

Histological findings on ileal section stained with $\mathrm{H} \& \mathrm{E}$ of a control rats illustrate a normal mucosa with prominent villus, and thus showed nil Chiu score, whereas, the ileal section of IIRI-induced rats was characterized by the presence of denuded villi and lamina propria with massive infiltration of neutrophils and extensive necrosis accompanied by disrupted brush border and edema. These pathological changes were produced the highest Chiu score due to increased oxidative stress, an inflammatory response which results in increased intestinal permeability and eventually end up in extensive necrosis and edema (intestinal damage). The above results are in accord with the results of Yan et al [26]. The ileal section of SABpretreated rats revealed mild villi denudation with lesser neutrophil infiltration, edema and necrosis with lower Chiu score, thus indicating its gastroprotective activity. The ileal section of SAB control rats displayed normal morphology with prominent villi and appeared similar to control rats with nil Chiu score.

\section{CONCLUSION}

The finding of this study demonstrate that SAB protects the rat small intestine (ileum) by alleviating oxidative stress, inflammatory response, neutrophil infiltration, intestinal permeability, and histopathological changes. Hence, SAB has a potential for treating ischemic/reperfusion condition in combination with standard drugs. Nevertheless, further experiments are needed to evaluate the underlying mechanisms of its gastro-protective activity.

\section{DECLARATIONS}

\section{Acknowledgement}

The authors sincerely thank Nanchang University for funding this study (no. NCU-190904).

\section{Conflict of Interest}

No conflict of interest associated with this work. 


\section{Contribution of Authors}

The authors declare that this work was done by the authors named in this article and all liabilities pertaining to claims relating to the content of this article will be borne by them.

\section{Open Access}

This is an Open Access article that uses a funding model which does not charge readers or their institutions for access and distributed under the terms of the Creative Commons Attribution License (http://creativecommons.org/licenses/by/ 4.0) and the Budapest Open Access Initiative (http://www.budapestopenaccessinitiative.org/rea d), which permit unrestricted use, distribution, and reproduction in any medium, provided the original work is properly credited.

\section{REFERENCES}

1. Sun $Y, X u Y$, Wang $G N$. Pterostilbene prevents intestinal ischemia reperfusion injury in Wistar rats via modulation of antioxidant defense and inflammation. Trop $\mathrm{J}$ Pharm Res 2015; 14(8): 1383-1391.

2. Vollmar B, Menger MD. Intestinal ischemia/reperfusion: microcirculatory pathology and functional consequences. Langenbeck's Arch Surg 2011; 396(1): 13-29.

3. Li C, Li Q, Liu YY, Wang MX, Pan CS, Yan L, Chen YY, Fan JY, Han JY. Protective effects of Notoginsenoside $R 1$ on intestinal ischemia-reperfusion injury in rats. Am J Physiol Gastrointest Liver Physiol 2014; 306(2): G11122.

4. Yang X, Bai H, Cai W, Li J, Zhou Q, Wang Y, Han J, Zhu $X$, Dong $M, H u D$. Lycium barbarum polysaccharides reduce intestinal ischemia/reperfusion injuries in rats. Chem Biol Interact 2013; 204(3): 166-172.

5. Impellizzeri $D$, Cordaro $M$, Campolo M, Gugliandolo E, Esposito E, Benedetto F, Cuzzocrea S, Navarra M. Antiinflammatory and antioxidant effects of flavonoid-rich fraction of bergamot juice (BJe) in a mouse model of intestinal ischemia/reperfusion injury. Front Pharmacol 2016; 7.

6. Okudan N, Belviranlı M, Gökbel H, Öz M, Kumak A. Protective effects of curcumin supplementation on intestinal ischemia reperfusion injury. Phytomed 2013, 20(10): 844-848.

7. Hou S, Zhao MM, Shen PP, Liu XP, Sun Y, Feng JC. Neuroprotective Effect of Salvianolic Acids against Cerebral Ischemia/Reperfusion Injury. Int J Mol Sci 2016; 17(7): 1190.

8. Qiao $Z, X U Y$ Y. Salvianolic acid b alleviating myocardium injury in ischemia reperfusion rats. Afr $J$ Tradit Complement Altern Med 2016; 13(4): 157-161.

9. Raoufi S, Baluchnejadmojarad T, Roghani M, Ghazanfari T, Khojasteh F, Mansouri M. Antidiabetic potential of salvianolic acid $B$ in multiple low-dose streptozotocininduced diabetes. Pharm Biol 2015; 53(12): 1803-1809.

10. Zhou QH, Wang HM. Protective effect of Salvia miltiorrhiza on gastric mucosa [J]. China J Modern Med 2008; 5: 022

11. Fu J, Fan HB, Guo Z, Wang Z, Li XD, Li J, Pei GX. Salvianolic acid $B$ attenuates spinal cord ischemiareperfusion-induced neuronal injury and oxidative stress by activating the extracellular signal-regulated kinase pathway in rats. J Surg Res 2014; 188(1): 222-230.

12. Kong $R$, Gao $Y$, Sun $B$, Chen $H$, Wang $G$, Wang $X$, Zhu $H$, Pan $S$, Xue $D$, Jiang $H$. The strategy of combined ischemia preconditioning and salvianolic acid- $B$ pretreatment to prevent hepatic ischemia-reperfusion injury in rats. Dig Dis Sci 2009; 54(12): 2568-2576.

13. NRC [National Research Council]. 1996. Guide for the Care and Use of Laboratory Animals. 7th ed. Washington DC: National Academy Press

14. Chang $C L$, Sung $P H$, Sun $C K$, Chen $C H$, Chiang $H J$, Huang TH, Chen YL, Zhen YY, Chai HT, Chung SY, Tong MS. Protective effect of melatonin-supported adipose derived mesenchymal stem cells against small bowel ischemia-reperfusion injury in rat. $J$ pineal Res 2015; 59(2): 206-220.

15. Chiu CJ, McArdle AH, Brown R, Scott HJ, Gurd FN. Intestinal mucosal lesion in low-flow states: I. A morphological, hemodynamic, and metabolic reappraisal. Arch Surg 1970; 101(4): 478-483.

16. Buchholz B, Kaczorowski DJ, Sugimoto $R$, Yang $R$, Wang Y, Billiar TR, McCurry KR, Bauer AJ, Nakao A. Hydrogen inhalation ameliorates oxidative stress in transplantation induced intestinal graft injury. $A m \mathrm{~J}$ Transplant 2008; 8(10): 2015-2024.

17. Liu XH, Yang YW, Dai HT, Cai SW, Chen RH, Ye ZQ. Protective role of adiponectin in a rat model of intestinal ischemia reperfusion injury. World J Gastroenterol 2015; 21(47): 13250

18. Liu CS, Chen NH, Zhang JT. Protection of PC12 cells from hydrogen peroxide-induced cytotoxicity by salvianolic acid $B$, a new compound isolated from Radix Salviae miltiorrhizae. Phytomed 2007; 14(7): 492-497.

19. Tongqiang L, Shaopeng L, Xiaofang $Y$, Nana S, Xialian $X$, Jiachang $H$, Ting $Z$, Xiaoqiang $D$. Salvianolic acid $B$ prevents iodinated contrast media-induced acute renal injury in rats via the PI3K/Akt/Nrf2 pathway. Oxi Med Cell Longev 2016; 2016

20. Jiang YF, Liu ZQ, Cui W, Zhang WT, Gong JP, Wang $X M$, Zhang $Y$, Yang MJ. Antioxidant effect of salvianolic acid $B$ on hippocampal CA1 neurons in mice with cerebral ischemia and reperfusion injury. Chin $\mathrm{J}$ Integrat Med 2015; 21: 516-522

21. Zhao GR, Zhang HM, Ye TX, Xiang ZJ, Yuan YJ, Guo $Z X$, Zhao LB. Characterization of the radical scavenging and antioxidant activities of danshensu and salvianolic acid B. Food Chem Toxicol 2008; 46(1): 73-81.

22. Yang F, Chen Z, Lian Z, Guo Y, Liu G, Dong G. Inhibitory effects of salvianolic acid $B$ on myocardial cellular nuclear transfer of NF-KB p65 and the expression of

Trop J Pharm Res, October 2017; 16(10): 2437 
TNF- $\alpha$ during ischemia-reperfusion in rabbits hearts in vivo. China J Modern Med 2009; 5: 010.

23. Wang Y, Chen G, Yu X, Li Y, Zhang L, He Z, Zhang N, Yang $X$, Zhao $Y$, Li N, Qiu H. Salvianolic acid $B$ ameliorates cerebral ischemia/reperfusion injury through inhibiting TLR4/MyD88 signaling pathway. Inflamm 2016; 39(4): 1503-1513.

24. Shen $Z Y$, Zhang J, Song HL, Zheng WP. Bone-marrow mesenchymal stem cells reduce rat intestinal ischemiareperfusion injury, ZO-1 downregulation and tight junction disruption via a TNF- $\alpha$-regulated mechanism. World J Gastroenterol 2013; 19(23): 3583-3595.

25. Deng $Y$, Yang $M, X u F$, Zhang $Q$, Zhao $Q, Y u H$, Li D, Zhang G, Lu A, Cho K, Teng F. Combined salvianolic acid $B$ and ginsenoside $R g 1$ exerts cardioprotection against ischemia/reperfusion injury in rats. PloS One. 2015; 10(8): e0135435.

26. Yan L, Wu CR, Wang C, Yang CH, Tong GZ, Tang JG. Effect of Candida albicans on Intestinal Ischemiareperfusion Injury in Rats. Chin Med J 2016; 129(14): 1711. 